\title{
DISPLACEMENT FROM THE APPARENT VERTICAL IN FREE FALL.
}

\author{
By Will C. BAKER.
}

SyNOPSIS.

\begin{abstract}
A simple method is given of obtaining the displacement by operating in fixed space. It is simple and permits one to visualize the southward component. An appreciable difference in the result is obtained according as the trajectory is treated as a parabola or an ellipse, the expression found in the latter case being only two thirds that found for the former.
\end{abstract}

IN looking over the various treatments of the equatorward deflection of a freely falling body, the method herein described has not been found. It brings into prominence certain features that are perhaps worthy of record. The case dealt with is that of a body falling towards a homogeneous spherical earth that revolves uniformly about a fixed axis. These assumptions are common to most of the methods found, very few taking account of the earth's equatorial protuberance.

Let the figure represent a sphere of radius $R$, fixed in space; and let the earth be represented by a second sphere, coincident with the fixed sphere at time $t=0$, but rotating about the axis $O C$, with the small uniform angular velocity $\omega . A F$ represents a material rod of height $h$, fixed in the rotating sphere at latitude $\lambda$, and normal to the surface at the point $A . \quad F D$ is a plumb-line that takes the position shown due to the fact that it is in uniform circular motion about $O C$. Its direction gives the apparent vertical at the point $A$, as it is at rest with regard to the rotating sphere. The bob $D$ is supposed to hang just above the spherical surface, and in its rotation to trace out the small circle $D B K$. Consider now a mass $m$ released from the point $F$ at time $t=0$, and being at the moment of release at rest with regard to $F$. In space this body has at time $t=0$ an initial velocity

$$
(R+h) \omega \cos \lambda
$$

along $F H$, which is normal to the plane of the circle $D A C$, and it will experience in space an acceleration $\bar{g}$ directly towards $O$. Thus its trajectory will lie in the plane $O F H M$ which is fixed in space and which cuts the fixed sphere in the great circle $A B L$. It will appear later, however, that the body under these conditions always falls on the portion $B L$. 
As the plumb-bob is in uniform circular motion in the small circle $D B K$, it has the normal accelerating force $m r \omega^{2}$, along the radius $r$ of that circle. This force is due to the resultant of the two forces acting on the bob, viz., (I) the tension of the cord $m g$, along $D F$, where $g$ is the apparent value of the acceleration due to gravity at the point $D$; and

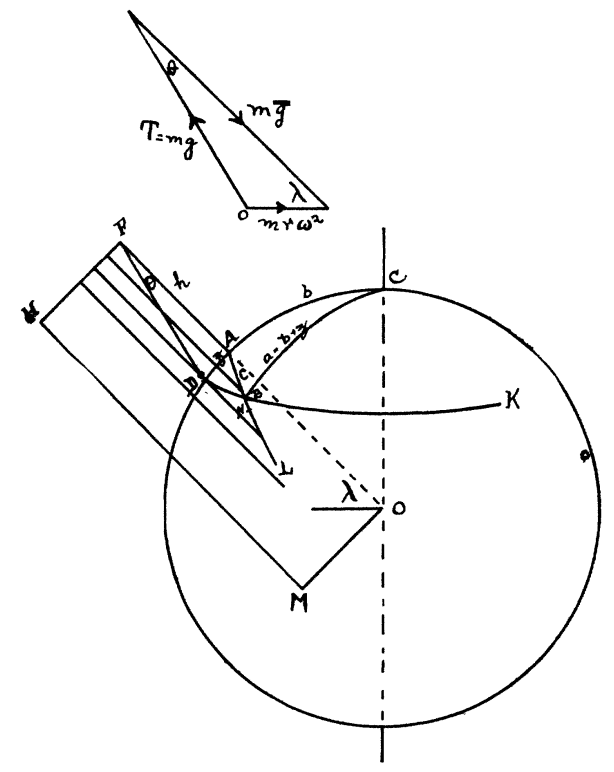

Fig. 1.

(2) its true weight $m \bar{g}$ along $D O$ where $m \bar{g}$ measures the force with which the bob is attracted towards the centre $O$. The relation of these vectors is shown in the upper part of the diagram. From this

or

$$
m g \sin \theta=m r \omega^{2} \sin \lambda
$$

$$
\begin{aligned}
\sin \theta & =\frac{r \omega^{2} \sin \lambda}{g} \\
& =\frac{R \cos \lambda \omega^{2} \sin \lambda}{g} .
\end{aligned}
$$

And, since $\theta$ is small, the $\operatorname{arc} z($ i.e., $A D)=h \sin \theta=\frac{h R \omega^{2} \sin \lambda \cos \lambda}{g}$. The angle $z$, subtended by the $\operatorname{arc} z$ at $O$, is given by

$$
\frac{h \omega^{2} \sin \lambda \cos \lambda}{g}
$$

and this has a maximum value for $\lambda=45^{\circ}$.

In the spherical triangle $A B C \cos a=\cos (b+z)=\cos c \cos b$ since the angle at $A=\pi / 2$, 


$$
\begin{aligned}
\cos c & =\frac{\cos (b+z)}{\cos b} \\
& =\cos z-\sin z \cot \lambda, \text { for } \cot \lambda=\tan b \\
& =\mathrm{I}-\frac{z^{2}}{2 !}+\frac{z^{4}}{4 !} \cdots-\left(z+\frac{z^{2}}{3 !} \cdots\right) \cot \lambda .
\end{aligned}
$$

Now even if we take $h$ as $40,000 \mathrm{~cm}$., which is the greatest value for which I can find ${ }^{1}$ any experiments, and if we consider these to have been made in the latitude of greatest southerly deviation $\left(45^{\circ}\right), z$ is found to be $1.07 \times 10^{-7}$ radians, and the terms in $z^{2}$ would appear only in the fourteenth place of decimals. Thus to this degree of approximation

$$
\cos c=\mathrm{I}-z \cos \lambda
$$

which on substituting the value of $z$ gives

but from the cosine series

$$
\cos c=\frac{\mathbf{I}-h \omega^{2} \cos ^{2} \lambda}{g}
$$

$$
\cos c=\mathrm{I}+\frac{c^{2}}{2 !}
$$

to terms in $c^{2}$, therefore on comparison

$$
c=\omega \sqrt{\frac{2 h}{g}} \cos \lambda
$$

and the arc $c$ (i.e., $A B)=R \omega \sqrt{\frac{2 h}{g}} \cos \lambda$, showing that $B$ is the point occupied by the plumb-bob at the instant that the falling body strikes the spherical surface (the angle $A B D$ being small, as it always is) for this is the angle that $D$ moves through in the time of fall $(\sqrt{2 h / g}$ when we neglect terms in $\omega^{2}$ ).

Consider next the trajectory in the fixed plane $O F H M$. It would appear at first sight that on account of the very small rotation of the earth during the fall no appreciable difference would result from treating this as a parabola or as an ellipse. If we denote the point of impact on the line $B L$ from a parabolic path as $N$, and that from an elliptical path as $N^{\prime}$ the two distances $A N$ and $A N^{\prime}$ will differ only by a very small part of either line, but when we realise that it is the difference $B N$ or $B N^{\prime}$ that we seek, and that $A B$ is nearly as long as $A N$ or as $A N^{\prime}$ it becomes evident that our result depends on the differences between quantities of the same order of magnitude, and that quantities otherwise insignificant become of importance. ${ }^{2}$

${ }^{1}$ Rundell, quoted by E. H. Hall, Phys. Rev., Vol. XVII., p. I86, I903.

${ }^{2}$ Even in the case of the extreme height quoted the distances are as follows: $A B=295955$ cm., $A N=295973 \mathrm{~cm}$., $A N^{\prime}=295967 \mathrm{~cm}$., differences of the order of $5 / 1000$ of a per cent. but $A N-A B=\mathrm{I} 8 \mathrm{~cm} ., A N^{\prime}-A B=\mathrm{I} 2 \mathrm{~cm}$. , and these differ by 66 per cent. 
Taking the line of action of the acceleration as constant, we have a constant horizontal velocity of $(R+h) \omega \cos \lambda$ during the fall $(\bar{t})$ and the resulting value of $A B$ will be $(R+h) \omega \bar{t} \cos \lambda$ and subtracting the value of $A B=\omega \bar{t} \cos \lambda$ we have

$$
\begin{aligned}
B N & =(R+h) \omega \bar{t} \cos \lambda-R \omega \bar{t} \cos \lambda \\
& =h \omega \bar{t} \cos \lambda \\
& =\frac{1}{2} \omega g \bar{t} \bar{t}^{3} \cos \lambda \text { since } h=\frac{1}{2} g t^{2} .
\end{aligned}
$$

This is of course along the great circle, and we get the easterly and the southerly displacements by multiplying this by the cosine and the sine of the angle $A B D$.

$$
\begin{aligned}
A B D & =\sin ^{-1} \frac{z}{A B} \\
& =\sin ^{-1} \frac{h R \omega^{2} \sin \lambda}{g(R+h) \omega t} \text { or very nearly, } \\
& =\sin ^{-1} \frac{h \omega \sin \lambda}{g \bar{t}} .
\end{aligned}
$$

Turning now to the elliptical path, take axes of $X$ and $Y$ respectively as $O F$ and $O M$. We have for the component acceleration parallel to $X$

$$
\frac{d^{2} x}{d t^{2}}=-\frac{g R^{2} x}{\left(x^{2}+y^{2}\right)^{3 / 2}},
$$

also the initial velocity along $F H$

Writing

$$
\left(\frac{d x}{d t}\right)_{0}=(R+h) \omega \cos \lambda
$$

we get

$$
x=x_{0}+\left(\frac{d x}{d t}\right)_{0} t+\left(\frac{d^{2} x}{d t^{2}}\right)_{0} t^{2}+\left(\frac{d^{3} x}{d t^{3}}\right)_{0} t^{3} \cdots
$$

$$
\bar{x}=(R+h) \omega \bar{t} \cos \lambda-\frac{g \omega \bar{t}^{3} \cos \lambda}{6}
$$

where $\bar{x}$ and $\bar{t}$ indicate the values of these two quantities at the instant of impact. Now treating this value $\left(B N^{\prime}=\bar{x}\right)$ as in the case of the parabolic path we find for $B N^{\prime}$

$$
\frac{1}{3} \omega g \bar{t}^{3} \cos \lambda
$$

which is the Gaussian expression usually obtained by a transformation to rotating axes. It differs from the value obtained on the parabolic path simply by the factor two thirds as explained above.

Physical Laboratory,

QUEEN'S UNIVERSITY,

Kingston, ONT.,

May 22, 1919. 